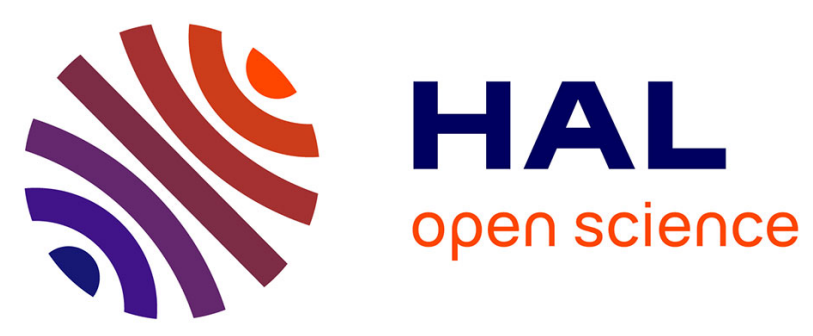

\title{
Quantitative analysis of lead position vs. correction of electrical dyssynchrony in an experimental model of LBBB/CRT
}

David Soto-Iglesias, Nicolas Duchateau, Constantine Butakoff, David Andreu, Juan Fernández-Armenta, Bart Bijnens, Antonio Berruezo, Marta Sitges,

Oscar Camara

\section{To cite this version:}

David Soto-Iglesias, Nicolas Duchateau, Constantine Butakoff, David Andreu, Juan FernándezArmenta, et al.. Quantitative analysis of lead position vs. correction of electrical dyssynchrony in an experimental model of LBBB/CRT. FIMH: Functional Imaging and Modeling of the Heart, Jun 2015, Maastricht, Netherlands. pp.74-82, 10.1007/978-3-319-20309-6_9 . hal-01208006

\section{HAL Id: hal-01208006 https://hal.inria.fr/hal-01208006}

Submitted on 1 Oct 2015

HAL is a multi-disciplinary open access archive for the deposit and dissemination of scientific research documents, whether they are published or not. The documents may come from teaching and research institutions in France or abroad, or from public or private research centers.
L'archive ouverte pluridisciplinaire HAL, est destinée au dépôt et à la diffusion de documents scientifiques de niveau recherche, publiés ou non, émanant des établissements d'enseignement et de recherche français ou étrangers, des laboratoires publics ou privés. 


\title{
Quantitative analysis of lead position vs. correction of electrical dyssynchrony in an experimental model of LBBB/CRT
}

\author{
David Soto-Iglesias $^{1 \star}$, Nicolas Duchateau ${ }^{3}$, Constantine Butakoff ${ }^{1}$, David \\ Andreu $^{2}$, Juan Fernández-Armenta ${ }^{2}$, Bart Bijnens ${ }^{1}$, Antonio Berruezo ${ }^{2}$, Marta \\ Sitges $^{2}$, and Oscar Camara ${ }^{1}$ \\ ${ }^{1}$ PhySense, DTIC, Universitat Pompeu Fabra, Barcelona, Spain, \\ ${ }^{2}$ Cardiology Department, Thorax Institute, Hospital Clinic, Barcelona, Spain \\ 3 Asclepios Research project, Inria Sophia Antipolis, France
}

\begin{abstract}
Cardiac resynchronization therapy (CRT) is a recommended treatment in patients with electrical dyssynchrony such as left bundle branch block (LBBB). The determination of the optimal leads position, and the quantification of the changes in electrical activation are two current major challenges. In this paper, we investigate these aspects through electroanatomical data from a controlled experimental protocol, which studied pigs with no structural disease under LBBB and CRT conditions. We propose to use a quasi-conformal mapping technique to standardize electroanatomical maps of endo- and epi-cardial walls of both ventricles to a common reference geometry, in which simple quantitative indices can be computed. Then, we investigate the relation between leads and simple surrogates of the recovery of the electrical activation based either on total activation times or on the spatial distribution of the patterns. Our methodology allows a better understanding of the complex electrical activation patterns in LBBB and CRT, and confirms hypotheses about the optimal leads position from previous studies.
\end{abstract}

Key words: Electro-anatomical mapping, LBBB, CRT.

\section{Introduction}

Cardiac resynchronization therapy (CRT) is a treatment used to improve cardiac pump function in patients with heart failure, by correcting the electrical activation of the heart and then making it contract synchronously. However, the high rate of non-responders, around $30 \%$, and the associated cost are still issues of primary concern [1]. For CRT optimization some studies aimed at identifying the best placement of the CRT leads, but results are contradictory: while one study suggested to avoid apical locations [2], others reported no significant differences [3,4]. A recent study [5] evaluated the optimal location for the left ventricle (LV) lead through in-silico experiments. Its results suggest that the

\footnotetext{
* david.soto@upf.edu
} 
LV lead should be placed distant from both the septum and scar regions. Further comparisons [6] between in-silico, experimental models and real data also confirmed that biventricular pacing is useful for recovering the electrical dyssynchrony between RV and LV ventricles. Despite these advances, the optimal lead placement is still highly controversial.

Electro-anatomical maps (EAMs) acquired with electrophysiological mapping systems are well established into the daily clinical routine for guiding radio-frequency ablation therapies e.g., in the treatment of different arrhythmias. EAMs provide local electrical activation information fused with a 3D representation of the anatomical area that is mapped. This information helps improving the understanding of the electrical activation patterns in different cardiac pathologies and subsequent treatment, such as left bundle branch block (LBBB) and CRT pacing, through subject-specific observations. Nevertheless EAMs are difficult to interpret for non-experienced observers. Moreover, it is not straighforward to compare, quantify and perform population-based statistics with data corresponding to different subjects or at different stages of the disease.

Several researchers have recently proposed mapping techniques to construct 2D reference systems for different organs such as the left ventricle [7], the atria [8], vertebral bones [9] or faces [10]. In this paper, we use a quasi-conformal mapping technique that allows creating a $2 \mathrm{D}$ reference map for EAMs of the endocardial and epicardial walls of the heart, including both ventricles. The approach is inspired from previous works [11] that used it in an experimental swine model of LBBB-induced electrical dyssynchrony, implanted with a CRT device. However, such works only considered the LV endocardium. In the present study, the joint analysis of the endo- and epi-cardial walls allows understanding aspects of the transmurality of the electrical activation. Furthermore, in order to quantitatively interpret these data, we propose a set of simple indices directly measured from the activation maps. Through these indices, we provide insights into the links between lead location and the correction of electrical dyssynchrony, which confirm previous hypotheses about the optimal leads location.

\section{Materials}

\subsection{Protocol}

A total of 9 pigs (median weight of $34 \mathrm{~kg}$ ) were studied from a dataset described in [12]. Animal handling was approved by the Ethics Committee at Anonymous hospital and conformed to international guidelines [12].

LBBB was induced in all animals using radio-frequency ablation, assisted by high rate pacing (160bpm) during the burning process to prevent from ventricular fibrillation. Further details about the protocol can be found in [12]. A CRT device (bipolar right ventricle (RV) lead, model Beflex RF45; Sorin Group) was then positioned in the animal model: the RV lead was placed at the apex while the LV lead was positioned through a lateral coronary vein or through a lateral or anterolateral position of the LV epicardial surface, depending on the accessibility of the animal's anatomy. 


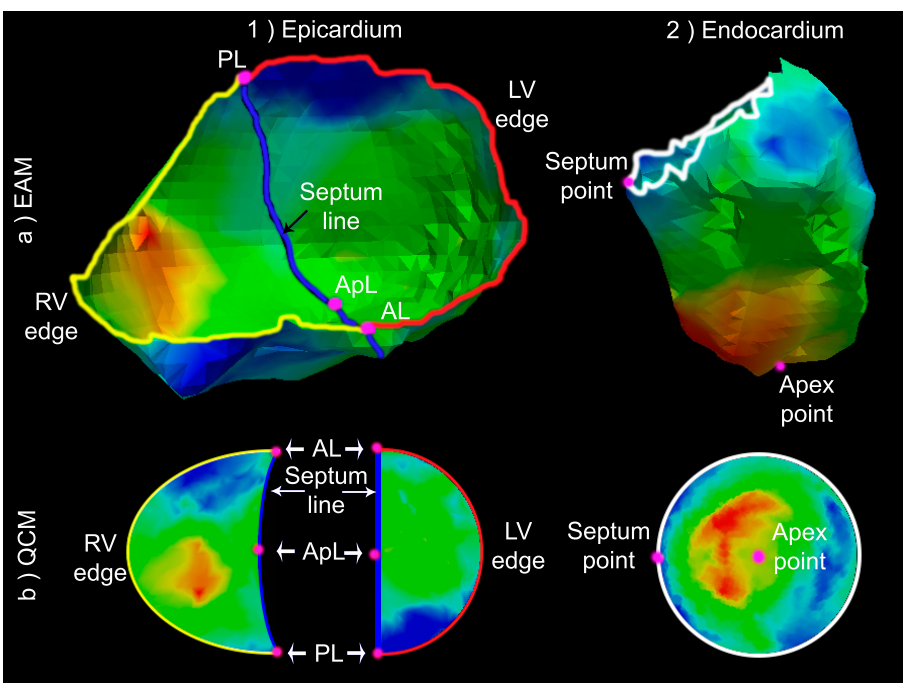

Fig. 1. 1) Epicardial EAM and reference 2D space. Septum line, anterior, (AL), apical $(\mathrm{ApL})$ and posterior (PL) landmarks, respectively. 2) Endocardial EAM and reference 2D space.

\subsection{Acquisition of electroanatomical maps (EAMs)}

Contact mapping data was collected with an electroanatomical mapping device (CARTO-XP, Biosense Webster, Diamond Bar, CA) after introducing a 3.5-mm tip catheter (Thermo-Cool Navi-Star, Biosense Webster) through the femoral artery until reaching the endocardium of the LV by the retrograde aortic access. Then, using the same catheter and system, an epicardial map of the LV and RV was performed.

EAMs were subsequently generated by reconstructing information from the set of sparse acquisition points, where the clinician placed the catheter to measure the electrical activity (around $2500 \mathrm{~ms}$ at $1 \mathrm{kHz}$ ). These measurements were processed and rendered in 3D to display the Local Activation Time (LAT), which is the parameter retained in this study to investigate electrical activation patterns. All maps were acquired on the day of the experiment, at baseline, postablation (LBBB) and with CRT pacing. The average number of acquired points were $171 \pm 66$ and $255 \pm 76$, for endocardium and epicardium, respectively.

\section{Methods}

We introduce a two-step approach to jointly analyze different EAMs looking for relations between the status of the heart, electrical dyssynchrony and CRT 
leads position. First, we construct a common reference 2D frame using a quasiconformal mapping. This has the advantage of standardizing both endo- and epicardial anatomies, thus facilitating the interpretation of corresponding EAMs. Then, we develop a set of both local and global quantitative measures to study standardized EAM data.

\subsection{Quasi-conformal mapping of endo- and epi-cardial walls}

In [11] we developed a quasi-conformal mapping (QCM) technique that takes advantage of the existence of an homeomorphism between the LV endocardial surface and a $2 \mathrm{D}$ disk. It was used to construct a reference common space for analyzing LV endocardial EAMs from different subjects. Here, we extend this technique to also incorporate information about the epicardium, i.e. including the external wall of the left and right ventricles of the heart. As can be seen in Fig. 1, epicardial EAMs only provide information of the LV lateral wall and RV free wall. Thus data on the septal wall are missing.

Since, we want to compare endo- with epicardial walls, we should not map the epicardium to a disk as in the LV endocardium data. Instead, we map the LV epicardium to the right half of a $2 \mathrm{D}$ disk, respecting the 17 AHA segment definition, as shown in Fig. 1. For the RV epicardium, we present a half-moon shape reference space as previously defined in [13].

The QCM of both heart walls are then computed by forcing every vertex coordinate to have a vanishing Laplacian, as shown in [11]. For the epicardium we only need to set the landmarks that provide correspondences between the EAM data and the 2D reference space. First, we define the septum line that divides LV and RV over the epicardial EAM mesh. To do so, three landmarks are manually selected: one over the anterior edge $(\mathrm{AL})$ of the mesh (Fig. 1): one over the posterior (PL) one; and finally one in an apical (ApL) position. Then, we define the septum line in the 3D EAM mesh as the geodesic line over the mesh that joins these landmarks. In the $2 \mathrm{D}$ reference space, the anterior landmark is placed at the $(1,0)$ point and the posterior to the $(-1,0)$ one (see top and bottom points, respectively, in Fig. 1). Then the septum line is defined as a geodesic line linking these two landmarks, being the LV free wall mapped onto the contour of half disk. Finally, a similar approach is used for the RV epicardial mapping disk. It has the advantage of preserving relative distances between the boundary points at the original 3D EAM mesh.

\subsection{Local and global quantitative measures}

EAMs provide rich information of electrical patterns of the heart, such as the time when every region of the heart is activated (LAT). Nevertheless EAMs are often analyzed globally by computing the total activation time (time difference between the start and end of activation). There is a need for more local measures of EAMs to better characterize the underlying electrical patterns with different therapy conditions. Here, we propose to derive indices from histograms of the isochrones in EAMs to better quantify electrical dyssynchrony of the endocardial 
a ) Baseline

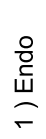

b ) LBBB

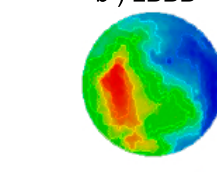

c ) CRT
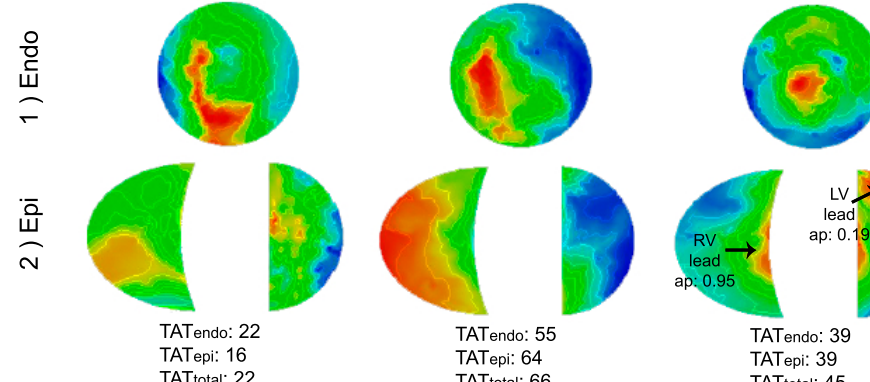

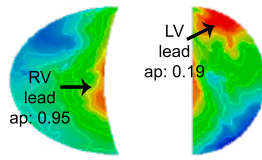

TATendo: 39 TATepi: 39

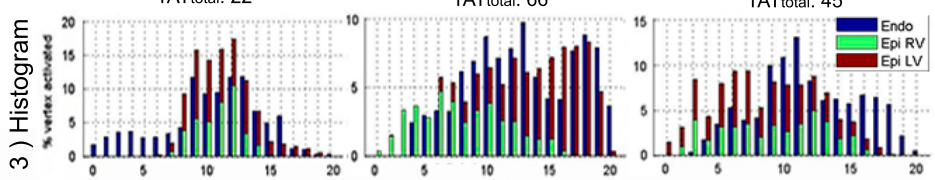

Fig. 2. Single case (\#2) from group 1 (basal LV lead + apical RV lead). Rows: QCM of endocardial and epicardial EAMs, and histogram of isochrones, at each stage.

LV free wall and the epicardium of both ventricles, as well as getting insights into the transmurality of the activation. Such indices are then compared to total activation times and to an apicality index representing lead position.

Total activation times (TATs). Since EAM data from both endo- and epicardial walls are already mapped onto the common reference space, it is straightforward to compute regional total activation times (for the LV, for the RV, for both ventricles) and to derive some indices comparing TATs at different stages of the experimental model. Therefore we define the following TAT-derived indices: the delay induced by LBBB is captured as the difference of the TATs between LBBB and baseline $\left(\Delta_{\text {base }}=T A T_{L B B B}-T A T_{\text {baseline }}\right)$; the effect of the CRT therapy is quantified as the difference of the TAT between LBBB and CRT $\left(\Delta_{C R T}=T A T_{L B B B}-T A T_{C R T}\right)$; and a recovery index expressing how close to the TAT after CRT is from its baseline value (Recovery $\left.=\left(\Delta_{\text {base }}-\Delta_{C R T}\right) * 100\right)$.

Indices from histograms of isochrones. The isochrones are defined for each EAM as the set of points with LATs within a certain range of the total TAT. The analysis of these isochrones can help on the interpretation of electrical patterns by studying the percentages of activated areas at a given timepoint. LAT values were previously normalized between 0 and $100 \%$ of the total TAT. Ranges of $5 \%$ are considered for the isochrones, corresponding to a total of 20 bins. An example of such histograms is shown in Fig. 2 and 3. 


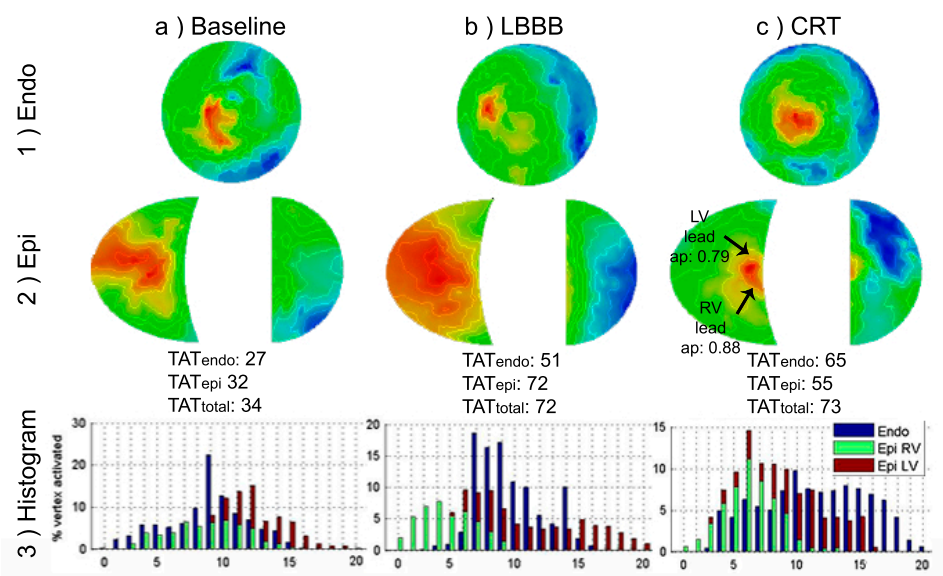

Fig. 3. Single case (\#9) from group 4 (basal LV lead + apical RV lead). Rows: QCM of endocardial and epicardial EAMs, and histogram of isochrones, at each stage.

Apicality index. For each lead, the apicality index is defined as: Apicality $=$ $1-r$, where $r$ is the distance between the lead and the center of the disk in polar coordinates. Leads position are identified from the CRT activation map using the k-means algorithm [14]. This served to identify the centers of two distinguishable clouds of activation within the initial $10 \mathrm{~ms}$ isochrones of the epicardium EAM, already mapped into the 2D common reference space. With this formulation, an apicality index closer to 1 or zero would mean an apical or basal lead, respectively.

\section{Results}

Between baseline and LBBB, the QRS width (measured on the ECG signals of the electro-anatomical system) significantly increased (median value from $55 \mathrm{~ms}$ to $84 \mathrm{~ms}$, respectively; $\mathrm{p}=0.005$ ).

The TAT values computed from the EAMs are summarized for each case in Table 1. Results are ordered according to four subgroups (G1-G4, different colours in Table 1). Such groups were created based on the apicality index, as follows: G1 (purple), basal LV lead and apical RV lead; G2 (red), mid LV lead and apical RV lead; G3 (green), both mid $L V$ and RV leads; G4 (yellow), both apical $L V$ and $R V$ leads. The behaviour of a couple of cases belonging to groups G1 and G4 is illustrated in Figs. 2 and 3, respectively.

The Recovery index (Section 3.2), which is computed from TATs, was used to compare the outcome of these subgroups. Average recoveries for subgroups 1, 2 and 3 were $>23 \%, 17 \%,<16 \%$, respectively. The two animals in G4 had a recovery of $-2.63 \%$ and $47.62 \%$. The substantial difference in the recovery of G4 subjects, and the fact that the TAT of one of these animals lies within the range 
for G1, point out the limits of a global index such as TAT to quantify electrical recovery through CRT. In contrast, the simple isochrones indexes we propose partially exploit the spatial distribution of the activation patterns, which may help improving the assessment of electrical recovery.

An histogram of isochrones is computed for each endo- and epicardial EAM. Table 2 lists the bin number within which the electrical activation starts and finalizes for each EAMs at baseline, LBBB and CRT. Additionally, transmural and inter-ventricular differences between these bins are also shown. These difference columns provide insights into the restoration of the synchronization of RV and LV after CRT. Three subjects in G1 achieved a quasi-complete recovery in the initial and final bins, but the \#1 did not recover the final one. G3, we can observe that the LV and RV are not completely synchronized after CRT. G4, subject number \#8 improves its electrical synchronization, while subject \#9 does not completely. Figure 3 provides elements of explanation for these electrical behaviours: both LV and RV leads are at close locations at the apex of the RV, inducing that the RV epicardium is activated two bins before the LV one, and also finalizing before the activation. Differences with the electrical pattern of a positive responder from G1 are visible in Fig. 2.3.c.

\section{Discussion and conclusions}

The presented apicality index allows characterizing CRT leads positioning from EAM data. Our findings confirm the optimal positions suggested by previous studies [5], namely that optimal pacing sites should target one lead at the apex and the other lead distant from it. Global indices such as TATs seem limited for the assessment of electrical recovery, while simple indices that integrate the spatial distribution of the activation complement our understanding.

The histogram of isochrones introduced in this work allows a qualitative analysis of the electrical activity in both ventricles. Complementary information in Fig. 2 and Fig. 3 help identifying the LBBB-induced dyssynchrony, which is presented both transmurally (between LV endo- and epi-cardium) and intercavity (LV and RV epicardium) due to the LBBB. The correction resulting from CRT

Table 1. Endo- and epi-cardial TATs (ms) at each stage of the protocol. Leads configuration subgroups are highlighted by the color code: G1, purple; G2, red; G3, green; G4, yellow. Corresponding electrical recovery is also indicated.

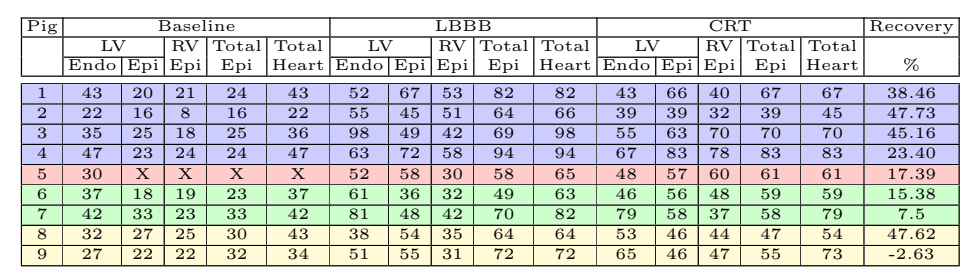


Table 2. Bin number corresponding to the isochrone within which the activation starts/ends, at baseline, LBBB and CRT. The two last columns indicate the differences between the initial and final number of $5 \%$ isochrones.

\begin{tabular}{|c|c|c|c|c|c|c|c|c|c|c|c|c|c|c|}
\hline \multirow[t]{2}{*}{ Pig } & \multicolumn{3}{|c|}{ Baseline } & \multicolumn{2}{|c|}{ Difference } & \multicolumn{3}{|c|}{ LBBB } & \multicolumn{2}{|c|}{ Difference } & \multicolumn{2}{|l|}{ CRT } & \multicolumn{2}{|c|}{ Difference } \\
\hline & \begin{tabular}{|l} 
I \\
Endo
\end{tabular} & V & $\begin{array}{l}\text { RV } \\
\text { Epi }\end{array}$ & $\begin{array}{c}\text { LV } \\
\text { Endo-Epi }\end{array}$ & $\begin{array}{c}\text { Epi } \\
\text { LV-RV }\end{array}$ & $\begin{array}{r}\text { LV } \\
\text { Endo } \\
\end{array}$ & VEpi & \begin{tabular}{|l} 
RV \\
Epi
\end{tabular} & $\begin{array}{c}\text { LV } \\
\text { Endo-Epi }\end{array}$ & $\begin{array}{c}\text { Epi } \\
\text { LV-RV }\end{array}$ & \begin{tabular}{|c|c|}
\multicolumn{2}{|c|}{ LV } \\
Endo
\end{tabular} & $\begin{array}{l}R V \\
\text { Epi } \\
\text { pi }\end{array}$ & \begin{tabular}{|c|} 
LV \\
Endo-Epi
\end{tabular} & $\begin{array}{c}\text { Epi } \\
\text { LV-RV }\end{array}$ \\
\hline 1 & $0 / 20$ & $3 / 13$ & $2 / 11$ & $3 /-7$ & $1 / 2$ & $5 / 18$ & $3 / 20$ & $0 / 13$ & $-2 / 2$ & $3 / 7$ & \begin{tabular}{|l|l|}
$2 / 16$ & $0 / 20$ \\
\end{tabular} & $0 / 12$ & $-2 / 4$ & $0 / 8$ \\
\hline$\frac{2}{2}$ & $0 / 20$ & $6 / 19$ & $7 / 14$ & $6 /-1$ & $-1 / 5$ & $3 / 20$ & $6 / 20$ & $0 / 16$ & $3 / 0$ & $6 / 4$ & \begin{tabular}{|l|l|}
$2 / 20$ & $0 / 17$ \\
\end{tabular} & $1 / 16$ & $-2 /-3$ & $-1 / 1$ \\
\hline 3 & $1 / 20$ & $1 / 14$ & $0 / 11$ & $0 /-4$ & $1 / 3$ & $0 / 20$ & $6 / 16$ & $\begin{array}{ll}1 / 9 \\
\end{array}$ & $6 /-4$ & $5 / 7$ & \begin{tabular}{|l|l|}
$5 / 20$ & $1 / 20$ \\
\end{tabular} & $0 / 20$ & $-4 / 0$ & $1 / 0$ \\
\hline 4 & $0 / 20$ & $6 / 16$ & $6 / 16$ & $6 /-4$ & $0 / 0$ & $3 / 17$ & $5 / 20$ & $0 / 11$ & $2 / 3$ & $5 / 9$ & \begin{tabular}{l|l|l|l|}
$3 / 19$ & $0 / 20$ \\
\end{tabular} & $0 / 18$ & $-3 / 1$ & $0 / 2$ \\
\hline 5 & $\mathrm{x}$ & $\mathrm{x}$ & $\mathrm{x}$ & $\mathrm{x}$ & $\mathrm{x}$ & $4 / 20$ & $0 / 18$ & $0 / 9$ & $-4 /-2$ & $0 / 9$ & \begin{tabular}{l|l|}
$2 / 20$ & $0 / 19$ \\
\end{tabular} & $0 / 20$ & $-2 /-1$ & $0 /-1$ \\
\hline 6 & $0 / 20$ & $10 / 18$ & $8 / 16$ & $10 /-2$ & $2 / 2$ & $1 / 20$ & $5 / 16$ & $0 / 11$ & $4 /-4$ & $5 / 5$ & \begin{tabular}{|l|l|}
$5 / 19$ & $0 / 20$ \\
\end{tabular} & $5 / 20$ & $-5 / 1$ & $-5 / 0$ \\
\hline 7 & $0 / 18$ & $3 / 20$ & $3 / 12$ & $3 / 2$ & $0 / 8$ & $0 / 20$ & $9 / 20$ & $3 / 13$ & $9 / 0$ & $6 / 7$ & \begin{tabular}{|l|l|}
$0 / 20$ & $2 / 13$ \\
\end{tabular} & $3 / 17$ & $2 /-7$ & $-1 /-4$ \\
\hline 8 & $0 / 15$ & $7 / 19$ & $9 / 20$ & $7 / 4$ & $-2 /-1$ & $7 / 18$ & $3 / 20$ & $0 / 11$ & $-4 / 2$ & $3 / 7$ & \begin{tabular}{|l|l|}
$0 / 20$ & $0 / 17$ \\
\end{tabular} & $0 / 16$ & $0 /-3$ & $0 / 1$ \\
\hline 9 & $0 / 15$ & $9 / 20$ & $2 / 14$ & $9 / 5$ & $7 / 6$ & $3 / 16$ & $5 / 20$ & $0 / 9$ & $2 / 4$ & $5 / 11$ & \begin{tabular}{|l|l|}
$2 / 20$ & $2 / 16$ \\
\end{tabular} & $0 / 13$ & $0 /-4$ & $2 / 3$ \\
\hline
\end{tabular}

can also be assessed. By looking at large changes in the slope of the histograms, the entrance onto the Cardiac Conduction System (CCS) via the Purkinje network can also be identified (e.g. in Fig. 3 the entrance onto the CCS is produced at the bin \#7 of the histogram).

One needs to be conscious of the sensibility of the method to the location of the landmarks used for the mapping, and the difficulty of acquiring EAMs in real patients with such a distributed sampling of the acquired points. However, the value of our study resides in allowing a better understanding of the complex mechanisms of electrical activation, in a LBBB/CRT protocol where external factors are controlled. The effect of damaged regions (e.g. regional infarct) on both the activation patterns and the optimal lead placement will be studied in further work.

\section{Acknowledgments}

This study was partially founded by the Spanish Ministry of Science and Innovation (TIN2011-28067) and the Spanish Industrial and Technological Development Center (cvREMOD-CEN-20091044). The authors gratefully acknowledge the support of N Solanes, M Rigol, E Silva, A Doltra, L Gabrielli, L Mont, J Brugada (Hospital Clnic, Barcelona, Spain) and A Barcelo (Sorin Group, Barcelona, Spain) on the experimental protocol.

\section{References}

1. Bleeker, G., et al: Clinical versus echocardiographic parameters to assess response to cardiac resynchronization therapy. Am J Cardiol 97 (2006) 260-263

2. Singh, J., et al: Left ventricular lead position and clinical outcome in the multicenter automatic defibrillator implantation trial-cardiac resynchronization therapy (MADIT-CRT) trial. Circulation 123 (2011) 1159-166

3. Gold, M., et al: Comparison of stimulation sites within left ventricular veins on the acute hemodynamic effects of cardiac resynchronization therapy. Heart Rhythm 2 (2005) 376-381 
4. Saxon, L., et al: Influence of left ventricular lead location on outcomes in the COMPANION study. J. Cardiovasc. Electrophysiol. 20 (2009) 764-768

5. Huntjens, P., et al: Influence of left ventricular lead position relative to scar location on response to cardiac resynchronization therapy: a model study. Europace 16 (2014) 62-68

6. Lumens, J., et al: Comparative electromechanical and hemodynamic effects of left ventricular and biventricular pacing in dyssynchronous heart failure. JACC 62 (2013) 2395-403

7. Soto-Iglesias, D., et al: Evaluation of different mapping techniques for the integration of electro-anatomical voltage and imaging data of the left ventricle. Funtional Imaging and modeling of the heart (FIMH'13) Lecture Notes in Computer Science (LNCS) 7945 (2013) 391-399

8. Karim, R., et al: Surface flattening of the human left atrium and proof-of-concept clinical applications. Computerized Medical Imaging and Graphics 38(4) (2014) $251-266$

9. Lam, K.C., et al: Genus-one surface registration via Teichmüller extremal mapping. MICCAI 3 (2014) 25-32

10. Zeng, W., et al: Ricci flow for 3D shape analysis. IEEE PAMI 32 (2010) 662-677

11. Soto-Iglesias, D., et al: Analyzing electrical patterns in an experimental swine model of dyssynchrony and CRT. IEEE Computing in Cardiology (CINC'13) 40 (2013) 623-626

12. Rigol, M., et al: Development of a swine model of left bundle branch block for experimental studies of cardiac resynchronization therapy. J. Cardiovascular Translational In press (2013)

13. Jing, L., et al: Patients with repaired tetralogy of Fallot suffer from intra- and inter-ventricular cardiac dyssynchrony: a cardiac magnetic resonance study. J Cardiovasc. Imaging 12 (2014) 1333-343

14. MacQueen, J.: Some methods for classification and analysis of multivariate observations. Proceedings of the Fifth BSMSP, Volume 1: Statistics (1967) 281-297 